\title{
Técnicas projetivas na avaliação de aspectos psicopatológicos da anorexia e bulimia
}

\author{
Rodrigo Sanches Peres ${ }^{1}$ - Universidade Federal de Uberlândia, Uberlândia, Brasil \\ Manoel Antônio dos Santos - Universidade de São Paulo, Ribeirão Preto, Brasil
}

\begin{abstract}
Resumo
O presente estudo teve como objetivo verificar a utilização de técnicas projetivas na avaliação de características psicopatológicas de pacientes com transtornos alimentares. Trata-se de uma revisão sistemática de literatura, realizada a partir de buscas nas bases de dados LILACS e Banco de Teses/CAPES com o emprego dos descritores "anorexia" e "bulimia". As referências localizadas foram selecionadas, recuperadas na íntegra e submetidas a uma apreciação qualitativa, organizada em dimensões de análise específicas. As principais características psicopatológicas reportadas foram: passividade, introversão e obsessividade, conflitos, tensões e angústias em relação à figura materna, grave distorção da imagem corporal e tendência à fusão nos relacionamentos interpessoais. Evidenciou-se, ainda, o predomínio de delineamentos metodológicos incipientes. Novas pesquisas envolvendo o emprego de técnicas projetivas no contexto dos transtornos alimentares são necessárias para prover os profissionais de evidências empíricas, que possam ser aproveitadas em protocolos de avaliação multidisciplinar adequados à população brasileira.

Palavras-chave: Transtornos alimentares, Anorexia nervosa, Bulimia nervosa, Técnicas projetivas, Instrumentos de avaliação.
\end{abstract}

Projective techniques in the assessment of psychopathology of patients with anorexia and bulimia

\begin{abstract}
This study aimed to review the Brazilian literature on the use of projective techniques in the assessment of psychopathological features of patients with eating disorders. It is a systematic literature review from searches on LILACS and CAPES Thesis Databases, with the use of the descriptors "anorexia" and "bulimia". The obtained references were selected and, later, recovered in full version and submitted to a qualitative appreciation, organized in specific dimensions. The main psychopathological features reported were: passivity, introversion and obsessiveness, conflicts, tensions and anxieties in relation to the maternal figure, severe distortion of body image and tendency to merge in interpersonal relationships. The researches analysis revealed predominance of weak methodological designs. New researches involving the use of projective techniques in the context of anorexia and bulimia are needed to provide practitioners with empirical evidence, which may be engaged in multidisciplinary assessment protocols adapted to the Brazilian population.

Keywords: Eating disorders, Nervous anorexia, Nervous bulimia, Projective techniques, Assessment scales.
\end{abstract}

De acordo com Cordás e Neves (1999), diversos instrumentos de avaliação - tais como o Eating Attitudes Test (EAT), o Eating Disorder Inventory (EDI) e o Body Shape Questionnaire (BSQ) - foram criados a partir da década de 1980 com o intuito de subsidiar a exploração de aspectos psicopatológicos dos transtornos alimentares (TA), quadros clínicos que incluem a anorexia e a bulimia nervosas. Embora se apoiem, em maior ou menor grau, em conceitos concernentes à Psicologia, nenhum desses instrumentos pode ser designado como teste psicológico. Ocorre que essa designação se aplica apenas a instrumentos que avaliam características ou processos psicológicos e que, como salientam Werlang, Villemor-Amaral e Nascimento (2010), são fundamentados em um referencial teórico específico, atendem a requisitos psicométricos de validade e precisão e são referenciados a normas de padronização.

Não há, atualmente, qualquer teste psicológico considerado em condições de uso no país, nos termos

${ }^{1}$ Endereço para correspondência:

Universidade Federal de Uberlândia - Instituto de Psicologia

Avenida Pará, 1.720 - Bloco 2C - Campus Umuarama

38405-320 - Uberlândia-MG

E-mail: rodrigosanchesperes@yahoo.com.br da legislação vigente (Conselho Federal de Psicologia, 2003) - ou seja, dotado de qualidades psicométricas comprovadas mediante parecer favorável no Sistema de Avaliação de Testes Psicológicos (SATEPSI), que tenha sido criado especialmente para ser aplicado em pacientes com anorexia ou bulimia. Contudo, esse fato não representa um impedimento, uma vez que, de acordo com Freitas, Gorenstein e Appolinário (2002), a avaliação no contexto dos TA deve contemplar também seus aspectos psicopatológicos gerais e não se limitar a suas características psicopatológicas específicas.

Publicações anteriores (Campana, Campana \& Tavares, 2009; Cordás \& Neves, 1999; Freitas, Gorenstein \& Appolinário, 2002) discutem os alcances e limites de escalas, inventários e questionários que não são designados como testes psicológicos, mas que são passíveis de utilização, no meio brasileiro, junto a pacientes com anorexia ou bulimia. Esses instrumentos de avaliação, quando se almeja operacionalizar uma abordagem integrada, podem ser empregados em conjunto com testes psicológicos. Porém, consultas preliminares a periódicos disponíveis em bases de dados e livros nacionais revelaram que nenhuma publicação se dedica, especificamente, a uma apreciação dos testes psicológicos capazes de, no 
âmbito brasileiro, se prestar a essa finalidade de modo a compor um protocolo de avaliação multidisciplinar ${ }^{1}$.

Faz-se necessário, portanto, um mapeamento das pesquisas nacionais realizadas mediante a utilização de testes psicológicos junto a pacientes com anorexia ou bulimia para que se possa delinear, com base em evidências empíricas robustas, quais deles têm se revelado potencialmente proveitosos ante as especificidades dessa população. Para viabilizar maior aprofundamento, um mapeamento dessa natureza poderia contemplar, mais especificamente, os testes projetivos agrupados sob a rubrica das técnicas projetivas. Ocorre que, em virtude da etiologia multifatorial da anorexia e da bulimia, é imprescindível uma avaliação aprofundada de seus aspectos psicopatológicos. $\mathrm{E}$ as técnicas projetivas têm potencial de oferecer importantes contribuições nesse sentido.

Afinal, conforme Chabert (2004), as técnicas projetivas, por serem historicamente atreladas ao referencial psicanalítico, se dedicam à investigação dos processos mentais profundos e inconscientes e se caracterizam por permitirem ao sujeito ampla liberdade de expressão e por exigirem um intenso grau de criação e elaboração pessoal. Isso se deve ao fato de veicularem estímulos pouco ou nada estruturados, que fomentam o processo de projeção - ou seja, o fenômeno psíquico que consiste na exteriorização de conteúdos internos - e favorecerem a emissão de respostas livres, capazes de revelar a maneira própria do indivíduo perceber, sentir e interpretar a situação (Güntert, 2000).

Tendo em vista o que precede, o presente estudo teve como objetivo verificar a utilização dos testes psicológicos agrupados sob a rubrica das técnicas projetivas na avaliação de características psicopatológicas associadas aos TA em pesquisas brasileiras na última década (2000-2010). Tal enfoque se justifica levando-se em consideração que as revisões sistemáticas de literatura científica possuem um inegável valor prático, uma vez que facilitam o acesso aos resultados obtidos por um conjunto de pesquisadores relativos a um determinado tema $e$, consequentemente, permitem a identificação tanto de lacunas, cujo preenchimento se faz necessário, quanto de repetições que devem ser evitadas em novas pesquisas.

\section{Método}

Para a localização dos estudos que interessavam ao objetivo definido, foram realizadas, mediante o emprego dos descritores "anorexia" e "bulimia", buscas em diferentes bases de dados on-line. Optou-se por adotar tal estratégia metodológica tendo- se em vista que a divulgação científica pela internet, mais ágil e menos onerosa, tem sido cada vez mais valorizada nos dias de hoje. A combinação de bases de dados foi levada em consideração por representar a alternativa mais eficiente para um levantamento bibliográfico completo. Partindo desse princípio, foram consultadas duas bases de dados nacionais distintas: LILACS e Banco de Teses/CAPES. Com isso, tornouse possível localizar tanto artigos publicados em revistas científicas quanto teses ou dissertações defendidas no país referentes ao assunto em pauta.

Os resumos das referências localizadas com esse procedimento foram submetidos a uma leitura preliminar, cujo propósito básico foi determinar quais delas eram efetivamente pertinentes ao presente estudo. A seguir, as referências selecionadas foram recuperadas na íntegra e submetidas a uma apreciação qualitativa, por meio do preenchimento de um protocolo de análise, concebido especialmente para o presente estudo, com o propósito de facilitar a execução de leituras analíticas. Para melhor operacionalização, os achados oriundos dessas leituras foram organizados e tabulados em cinco dimensões de análise: a) objetivo; b) amostra/participantes; c) técnicas projetivas utilizadas; d) métodos de análise adotados e e) resultados.

Por fim, mostrou-se oportuno, diante do objetivo do presente estudo, tecer algumas considerações acerca de cada uma das técnicas projetivas adotadas nas pesquisas revisadas, destacando seus alcances e limites em termos teóricos e práticos, quando aplicadas, especificamente, no contexto dos TA. Ademais, optou-se por abordar dois aspectos metodológicos das pesquisas revisadas, os quais foram considerados particularmente problemáticos por razões que serão detalhadas adiante. São eles: a amplitude das amostras adotadas e os critérios de inclusão/exclusão estabelecidos para os participantes.

\section{Resultados}

As buscas executadas conduziram à localização de apenas cinco pesquisas nacionais publicadas na última década que foram realizadas mediante o emprego de técnicas projetivas junto a pacientes com anorexia e bulimia. A Tabela 1 apresenta a caracterização de cada uma delas quanto às dimensões de análise já mencionadas. Desse modo, ilustra que Sopezki (2007), adotou um método de análise do Psicodiagnóstico de Rorschach "método Klopfer" - que conta com parecer favorável no SATEPSI. Os resultados obtidos na pesquisa em questão, considerados em seu conjunto, reforçam a existência de um padrão relacional recorrente entre mulheres acometidas por transtornos alimentares. 
Tabela 1. Caracterização das Referências Revisadas quanto às Dimensões de Análise Estabelecidas

\begin{tabular}{|c|c|c|c|c|c|c|}
\hline $\begin{array}{c}\text { Autores e } \\
\text { ano do } \\
\text { artigo }\end{array}$ & Objetivo & Amostra/Participantes & Técnicas projetivas utilizadas & Método de análise & Resultados & Indicadores principais \\
\hline $\begin{array}{l}\text { Bronstein } \\
(2005)\end{array}$ & $\begin{array}{l}\text { Avaliação de aspectos da } \\
\text { cognição e da afetividade em } \\
\text { uma amostra de mulheres } \\
\text { com anorexia e bulimia }\end{array}$ & $\begin{array}{l}\text { Amostra clínica, constituída por } \\
\text { mulheres com anorexia e bulimia } \\
(\mathrm{n}=10) \text { em tratamento e amostra } \\
\text { não-clínica }(\mathrm{n}=10)\end{array}$ & $\begin{array}{l}\text { Teste de Apercepção Temática } \\
\text { (TAT) }\end{array}$ & "Método Telles" & $\begin{array}{l}\text { As integrantes da amostra clínica se caracterizavam por uma precária } \\
\text { diferenciação entre o mundo interno e o mundo externo. } \\
\text { Entretanto, apresentaram boa percepção da realidade em relação aos } \\
\text { estímulos evocados pelas pranchas do TAT, sendo, nesse aspecto, } \\
\text { semelhantes às integrantes da amostra não-clínica }\end{array}$ & Não informados \\
\hline $\begin{array}{l}\text { Oliveira e } \\
\text { Santos } \\
\text { (2006) }\end{array}$ & $\begin{array}{l}\text { Caracterização do perfil } \\
\text { psicológico em uma amostra } \\
\text { de pessoas do gênero } \\
\text { masculino e feminino com } \\
\text { anorexia e bulimia em } \\
\text { seguimento clínico }\end{array}$ & $\begin{array}{l}\text { Amostra clínica constituída por } \\
\text { pessoas de ambos os sexos, com } \\
\text { diagnóstico de bulimia }(\mathrm{n}=18) \text { e } \\
\text { anorexia ( } \mathrm{n}=11) \text {, em tratamento } \\
\text { junto a um serviço especializado }\end{array}$ & $\begin{array}{l}\text { Psicodiagnóstico de Rorschach, } \\
\text { Teste de Apercepção Temática } \\
\text { (TAT), House-Tree-Person (HTP), } \\
\text { Questionário Desiderativo } \\
\text { (QD) e Teste das Pirâmides } \\
\text { Coloridas de Pfister (TPC) }\end{array}$ & Não informados & $\begin{array}{l}\text { A maioria dos pacientes avaliados apresentava um controle } \\
\text { deficitário dos impulsos e conflitos importantes nos relacionamentos } \\
\text { com as figuras parentais, os quais se revelaram associados a intensos } \\
\text { desejos de aprovação social e consequentes temores de rejeição. } \\
\text { Distorções graves da autoimagem também foram observadas na } \\
\text { maioria dos participantes, em alguns casos em grau extremo, } \\
\text { evidenciando má formação ou dispersão dos limites da identidade. }\end{array}$ & Não informados ${ }^{2}$ \\
\hline $\begin{array}{l}\text { Silva e } \\
\text { Paegle } \\
\text { (2006) }\end{array}$ & $\begin{array}{l}\text { Verificação de indicadores } \\
\text { psicopatológicos, em termos } \\
\text { das relações objetais, em um } \\
\text { caso de anorexia }\end{array}$ & $\begin{array}{l}\text { Estudo de caso único, com } \\
\text { paciente com anorexia } \\
\text { tratamento em } \\
\text { psicoterapêutico psiquiátrico }\end{array}$ & $\begin{array}{l}\text { Teste de Relações Objetais } \\
\text { (TRO) e do Desenho da Figura } \\
\text { Humana (DFH) }\end{array}$ & $\begin{array}{l}\text { "Método Phillipson" } \\
\text { para análise do TRO e } \\
\text { método não- } \\
\text { padronizado para o } \\
\text { DFH, baseado na } \\
\text { impressão global das } \\
\text { produções }\end{array}$ & $\begin{array}{l}\text { Os resultados obtidos com o TRO apontaram uma significativa } \\
\text { diminuicão da capacidade de discriminacão e acentuada tendência à } \\
\text { fusão com objetos concretos nos relacionamentos interpessoais. Os } \\
\text { dados obtidos com o DFH revelaram que a paciente relutava em } \\
\text { estabelecer contatos mais íntimos nos relacionamentos interpessoais } \\
\text { e appresentava tendências agressivas orais. }\end{array}$ & $\begin{array}{l}\text { - dependência em relação aos } \\
\text { objetos evidenciada pelo uso } \\
\text { maciço de identificação projetiva } \\
\text { nas estórias } \\
\text { decorrentes das pranchas } 1 \text { e } 13 \\
\text { - indiferenciação entre realidade e } \\
\text { fantasia; fragmentação egóica nas } \\
\text { estórias das pranchas } 1,5 \text { e } 13 \\
\text { - pensamentos mágicos associados } \\
\text { a sentimentos de exclusão na } \\
\text { estória decorrente da prancha } 9 \\
\text { - incapacidade de reparação na } \\
\text { estória estimulada pela prancha } 11^{3} \\
\text { - figuras humanas representativas } \\
\text { de criancas }\end{array}$ \\
\hline $\begin{array}{l}\text { Peres e } \\
\text { Santos } \\
\text { (2006) }\end{array}$ & $\begin{array}{l}\text { Investigação da imagem } \\
\text { corporal em uma amostra de } \\
\text { mulheres com anorexia }\end{array}$ & $\begin{array}{l}\text { Amostra clínica constituída por } \\
\text { mulheres com anorexia }(\mathrm{n}=10) \text { em } \\
\text { tratamento }\end{array}$ & $\begin{array}{l}\text { Desenho da Figura Humana } \\
\text { (DFH) }\end{array}$ & $\begin{array}{l}\text { Método não- } \\
\text { padronizado, baseado } \\
\text { na avaliação de diversas } \\
\text { características formais e } \\
\text { de conteúdo das } \\
\text { produções }\end{array}$ & $\begin{array}{l}\text { A maioria das pacientes avaliadas possú́a uma acentuada fragilidade } \\
\text { egoica, caracterizava-se por uma marcante restrição do potencial } \\
\text { adaptativo, procurava controlar os próprios impulsos afetivos com } \\
\text { excessivo rigor e tendia à passividade, à introversão, à obsessividade } \\
\text { e à dependência. Parte delas apresentava uma imagem corporal } \\
\text { negativa, permeada claramente por sentimentos de inferioridade, } \\
\text { inadequação e insegurança. Em contrapartida, outras pacientes } \\
\text { revelaram uma imagem corporal idealizada, influenciada diretamente } \\
\text { por mecanismos de defesa compensatórios. }\end{array}$ & $\begin{array}{l}\text { - figuras humanas de tamanho } \\
\text { diminuto, pouco integradas e } \\
\text { pobres em detalhes } \\
\text { - figuras humanas com mãos } \\
\text { desconfiguradas } \\
\text { - figuras humanas com cabelos } \\
\text { escassos e desordenados } \\
\text { - figuras humanas com traços } \\
\text { faciais } \\
\text { simplificados excessivamente } \\
\text { - figuras humanas com correções e } \\
\text { retogues excessivos }\end{array}$ \\
\hline $\begin{array}{l}\text { Sopezki } \\
(2007)\end{array}$ & $\begin{array}{l}\text { Investigação da relação } \\
\text { primária mãe-filha em uma } \\
\text { amostra de mulheres com } \\
\text { anorexia e bulimia }\end{array}$ & $\begin{array}{l}\text { Amostra clínica constituída por } \\
\text { mulheres com anorexia }(\mathrm{n}=15) \text { e } \\
\text { bulimia }(\mathrm{n}=17) \text { em tratamento e } \\
\text { amostra não-clínica }(\mathrm{n}=19)\end{array}$ & Psicodiagnóstico de Rorschach & "método Klopfer" & $\begin{array}{l}\text { A capacidade de relacionamento afetivo-emocional das participantes } \\
\text { se encontrava preservada. Porém, foram constatadas, entre as } \\
\text { integrantes da amostra clínica, dificuldades importantes no manejo } \\
\text { de conflitos, tensões e angústias em relação à figura materna. }\end{array}$ & $\begin{array}{l}\text { frequência equivalente nas } \\
\text { amostras clínica e não-clínica dos } \\
\text { determinantes forma e cor } \\
\text { - frequência mais elevada entre as } \\
\text { mulheres com anorexia de } \\
\text { respostas de movimento } \\
\text { inanimado } \\
\text { - frequência mais elevada entre as } \\
\text { mulheres com bulimia de } \\
\text { fenômenos especiais (choque de } \\
\text { reação por dilatação, choque de } \\
\text { duração, choque acromático e } \\
\text { cromático, critica ao teste, choque } \\
\text { de exclamação e giro ansioso) }\end{array}$ \\
\hline
\end{tabular}


Já Bronstein (2005) optou por um método de análise do Teste de Apercepção Temática (TAT) "método Telles" - que, nos termos da legislação vigente, pode ser empregado somente para fins de pesquisa, não sendo autorizado no âmbito da prática profissional em Psicologia. A exemplo do Psicodiagnóstico de Rorschach, o TAT também admite a adoção de diversos métodos de análise. Contudo, nos dias de hoje, apenas o "método Murray" é dotado de qualidades psicométricas comprovadas mediante parecer favorável no SATEPSI.

Oliveira e Santos (2006) reportam à execução de um processo de avaliação psicológica no qual foram empregadas cinco técnicas projetivas. Tal pesquisa, contudo, não especifica os indicadores dos aspectos psicopatológicos observados. Ademais, não esclarece quais métodos de análise foram utilizados. Logo, não é possível delimitar as vantagens e desvantagens $\mathrm{da}$ aplicação de cada um deles no âmbito do protocolo de avaliação proposto. A despeito disso, os autores destacam que os aspectos psicopatológicos observados nos participantes não impossibilitam o estabelecimento de vínculos interpessoais, o que representa um indicador prognóstico favorável.

Silva e Paegle (2006) apresentam um estudo de caso de uma mulher com anorexia em psicoterapia, submetida à aplicação do Teste de Relações Objetais (TRO) e do Desenho da Figura Humana (DFH). Entretanto, o TRO não dispõe atualmente de nenhum método de análise adaptado para a população brasileira. Já o método de análise do DFH adotado não é padronizado, de modo que não conta com parecer favorável no SATEPSI. Não obstante, as autoras apontam que os dados oriundos da utilização das técnicas projetivas em questão foram determinantes para o manejo do caso.

Por fim, Peres e Santos (2006), na última das pesquisas revisadas para os fins do presente estudo, investigaram a imagem corporal de um grupo de mulheres com anorexia a partir do emprego do DFH. O método de análise adotado também não é padronizado. Porém, em contraste com aquele adotado por Silva e Paegle (2006), é baseado na avaliação de diversas características formais e de conteúdo dos desenhos, as quais foram interpretadas em relação a critérios de atribuição de significados estabelecidos pela literatura especializada.

\section{Discussão}

Conforme Chabert (2004), o Psicodiagnóstico de Rorschach viabiliza a identificação de indicadores psicopatológicos discretos, que podem passar despercebidos em uma entrevista clínica e apontar uma descompensação em via de desenvolvimento. Ademais, possibilita o delineamento de recursos adaptativos latentes, sobre os quais poderão se apoiar intervenções multidisciplinares. Logo, pode-se propor que a utilização dessa técnica projetiva no contexto dos TA tenda a ser potencialmente proveitosa, como, a propósito, apontam as duas pesquisas revisadas Oliveira e Santos (2006) e Sopezki (2007) - nas quais a mesma foi adotada.

Já o TAT possibilita o acesso a tendências inconscientes, que raramente são expressas em pensamentos (Murray, 2005). O medo mórbido de engordar e o controle obsessivo do peso corporal, fatores associados à anorexia e à bulimia, remetem justamente a essas tendências inconscientes. Nesse sentido, conclui-se que o mesmo é capaz, ao menos em tese, de fornecer elementos relevantes para o planejamento e o desenvolvimento da assistência multidisciplinar à população em questão. Os resultados obtidos por Bronstein (2005) reforçam essa proposição, mas possuem um alcance limitado, na medida em que decorrem da utilização de um método de análise do TAT que não conta com parecer favorável no SATEPSI.

Como mencionado, o HTP foi adotado apenas na pesquisa de Oliveira e Santos (2006). Trata-se de uma técnica de estimulação verbal e produção gráfica, pois emprega o desenho como principal veículo de projeção (Buck, 2003). Justamente nesse ponto reside sua principal vantagem, considerando-se que, tanto para Cunha (2000) quanto para Peres e Justo (2005), a comunicação por meio do grafismo é menos suscetível à utilização de defesas estereotipadas do que a linguagem verbal. Nesse sentido, o HTP é indicado especialmente para sujeitos que, como a maioria dos pacientes com anorexia ou bulimia, tendem ao retraimento.

Dentre os diversos métodos de análise do HTP existentes, apenas o "método Buck" conta atualmente com parecer favorável no SATEPSI. Na pesquisa de Oliveira e Santos (2006) não consta qual foi o método de análise do HTP escolhido. Contudo, deve-se mencionar que a obra na qual o "método Buck" é apresentada (Buck, 2003) não é citada entre as referências adotadas pelos autores. Ainda sobre a pesquisa em questão, vale mencionar que a mesma também recorreu ao Questionário Desiderativo (QD), técnica projetiva voltada à avaliação de defesas, impulsos, conflitos, recursos egoicos e relações objetais. O QD diferencia-se por ser uma técnica projetiva verbal tanto em termos da estimulação quanto da produção (Ocampo \& Arzeno, 1981a).

Ocorre que o sujeito recebe uma estimulação verbal, à qual deve responder, sem qualquer estímulo 
visual, também de forma verbal. Isso implica, conforme Nijamkin e Braude (2000), um maior esforço para a simbolização, o que torna o QD especialmente proveitoso no contexto dos TA, pois se sabe que aos mesmos geralmente se encontram associados comprometimentos dessa capacidade. Além disso, as referidas autoras defendem que o QD pode revelar traços de personalidade que, a exemplo do perfeccionismo e da obsessividade, tendem a potencializar o ideal de beleza associado à magreza que é subjacente à anorexia e à bulimia. Contudo, nenhum método de análise do QD se encontra atualmente adaptado para a população brasileira, o que, em última instância, inviabiliza a confirmação empírica dessa proposição.

Oliveira e Santos (2006) também empregaram o Teste das Pirâmides Coloridas (TPC), técnica projetiva de estimulação visual e produção verbal e motora que, para Justo e Van Kolck (1996), favorece a emergência de diferentes modalidades de reação do indivíduo e, assim, de distintas formas de expressão. Ademais, a tarefa proposta, por seu caráter lúdico, dificilmente é recusada, mesmo em se tratando de indivíduos, excessivamente retraídos, como o são muitos pacientes com TA ou b) que atravessam períodos de forte perturbação afetivo-emocional, a exemplo daqueles que se observam com muita frequência no curso da anorexia e da bulimia.

Em que pese a existência de diversos métodos de análise do TPC, apenas um deles - o "método Villemor-Amaral" - dispõe atualmente de parecer favorável no SATEPSI. É possível, com a utilização desse método, avaliar aspectos da dinâmica afetivoemocional e características das estruturas cognitivas do sujeito (Villemor-Amaral, 2005). $\mathrm{Na}$ pesquisa de Oliveira e Santos (2006) não consta qual método de análise do TPC foi selecionado. Uma obra do final da década de 1970 da autora do "método VillemorAmaral" consta das referências adotadas pelos autores. Entretanto, o referido método é apresentado, em sua versão vigente, em uma obra mais recente (VillemorAmaral, 2005).

Adotado por Silva e Paegle (2006), o TRO, como seu próprio nome esclarece, visa à avaliação das relações objetais, mas abrange também a exploração do sistema tensional inconsciente e o equilíbrio adaptativo do ego (Rosa \& Silva, 2005). A exemplo do Psicodiagnóstico de Rorschach e do TAT, o TRO é uma técnica projetiva de estimulação verbal e visual e produção verbal. Porém, veicula estímulos que variam significativamente em termos do conteúdo de realidade, de modo que, para Ocampo e Arzeno (1981b), apresenta um grau de estruturação intermediário. Por essa razão, o TRO mobiliza um clima emocional variado, possibilitando uma exploração minuciosa dos sentimentos experimentados na relação transferencial.

Esse é um de seus principais pontos positivos, pois as vivências do sujeito mobilizadas pela relação transferencial que se estabelece durante a aplicação de técnicas projetivas tendem a refletir as experiências emocionais referentes aos relacionamentos interpessoais internalizados em um sentido mais amplo. Partindo-se desse princípio e considerando-se que a qualidade do vínculo que se estabelece entre pacientes e profissionais de saúde é determinante para a adesão de pacientes com anorexia ou bulimia à assistência multidisciplinar, constata-se que o TRO também se destaca como uma técnica projetiva potencialmente proveitosa no contexto dos TA. Entretanto, o TRO não dispõe, atualmente, como mencionado, de nenhum método de análise adaptado para a população brasileira, o que restringe sua aplicabilidade.

Silva e Paegle (2006), assim como Peres e Santos (2006), também empregaram o DFH. Embora subsidie a avaliação da personalidade como um todo, essa técnica projetiva privilegia a imagem corporal, já que a tarefa proposta remete às concepções internalizadas que o sujeito tem de si mesmo (Peres, Santos \& Kruschewsky, 2007). É notório que uma marcante distorção da imagem corporal frequentemente representa um fator psicológico predisponente, precipitante e mantenedor da anorexia e da bulimia. Portanto, a avaliação da imagem corporal em pacientes com TA se afigura como um procedimento extremamente relevante. $\mathrm{O}$ mesmo, por extensão, poder-se-ia afirmar a propósito da aplicação do DFH.

Mas deve-se esclarecer que o DFH, em contraste com as demais técnicas projetivas mencionadas no presente estudo, pode ser empregado com duas finalidades distintas: a) avaliação da personalidade em crianças, adolescentes e adultos e b) avaliação da inteligência em crianças. Atualmente, dois métodos de análise do DFH se encontram adaptados à população brasileira: o "método Wechsler" e o "método Sisto". Ambos, porém, foram desenvolvidos para a avaliação da inteligência. Desse modo, os resultados oriundos das pesquisas tanto de Silva e Paegle (2006) quanto de Peres e Santos (2006), os quais apontam a pertinência do DFH no contexto dos TA, possuem um alcance limitado.

Por fim, é preciso contemplar dois aspectos metodológicos das pesquisas revisadas que se revelaram particularmente problemáticos. $\mathrm{O}$ primeiro desses aspectos é a amplitude das amostras. Ocorre que, em todas as pesquisas, as amostras foram 
constituídas por um número relativamente reduzido de pacientes. Possivelmente por essa razão, os dados reportados pela maioria delas - salvo a pesquisa de Sopezki (2007) - não foram submetidos a procedimentos estatísticos, os quais poderiam subsidiar a obtenção de resultados mais robustos. Sugere-se, portanto, que, em novas pesquisas, essa limitação seja superada por meio da utilização de amostras mais amplas.

O segundo aspecto metodológico se refere aos critérios de inclusão e exclusão adotados nas pesquisas revisadas. Ocorre que, exceto em Silva e Paegle (2006), não são fornecidas informações suficientes a esse respeito. Não se sabe, portanto, quais parâmetros foram privilegiados para a definição do diagnóstico de anorexia ou bulimia. Essa é uma questão relevante, tendo-se em vista que há variações entre os parâmetros definidos pelos sistemas de classificação de transtornos mentais vigentes. Talvez a principal delas seja em relação à distinção entre dois subtipos clínicos de anorexia - purgativo e restritivo - proposta pelo DSMIV, mas não adotada pela CID-10. Afinal, parece razoável propor que a cada um desses subtipos podem estar associados aspectos psicopatológicos específicos.

\section{Considerações finais}

O presente estudo aponta que ainda são escassas as pesquisas nacionais realizadas mediante $\mathrm{O}$ emprego de técnicas projetivas junto a pacientes com anorexia ou bulimia. As análises realizadas evidenciaram o predomínio de delineamentos metodológicos incipientes do ponto de vista da força das evidências proporcionadas pelos resultados reportados. Afinal, nas pesquisas revisadas foram adotadas amostras clínicas reduzidas, selecionadas com base em critérios diagnósticos que não são claramente reconhecidos.

A reversão dessa situação é necessária para que se possa determinar, com base em evidências empíricas, quais técnicas projetivas são de fato mais proveitosas ante as especificidades dessa população e que, desse modo, podem ser incorporadas a protocolos de avaliação multidisciplinares adequados ao meio brasileiro. Protocolos dessa natureza, ressalte-se, auxiliam a viabilizar, em uma abordagem integrada, uma melhor apreensão dos aspectos psicopatológicos gerais ou específicos da anorexia e bulimia, subsidiando, como consequência, o aperfeiçoamento da assistência multidisciplinar oferecida aos portadores de TA.

Por fim, é preciso esclarecer que o presente estudo, em decorrência da inexistência de outros semelhantes no meio nacional, proporciona uma visão panorâmica do assunto em pauta e contribui para levantar questões de natureza metodológica que podem nortear o desenvolvimento de novas pesquisas. Por outro lado, possui limitações, na medida em que contempla apenas a produção científica brasileira da última década. $O$ recorte temporal executado é coerente com uma opção metodológica adotada na maioria das revisões sistemáticas recentes, nas quais a literatura atual é privilegiada. Cotejá-la com a literatura nacional de décadas passadas ou com a literatura internacional foge ao escopo estabelecido, mas poderia resultar em novos e instigantes estudos, com objetivos distintos. Afinal, por se configurarem cada vez mais como um problema de saúde em escala mundial, os TA não representam uma preocupação atual e tampouco circunscrita ao país.

\section{Referências}

Bronstein, M. (2005). Os quadros anoréxicos: cognição, afetividade e realidade (Dissertação de Mestrado). São Paulo: Universidade de São Paulo - Instituto de Psicologia.

Buck, J. N. (2003). H-T-P: manual e guia de interpretação (R. C. Tardivo, trad.). São Paulo: Vetor.

Campana, A. N. N. B., Campana, M. B. \& Tavares, M. C. G. C. F. (2009). Escalas para a avaliação da imagem corporal nos transtornos alimentares no Brasil. Avaliação Psicológica, 8(3), 437-466.

Chabert, C. (2004). Psicanálise e métodos projetivos (A. J. Lelé \& E. M. A. C. Silva, trads.). São Paulo: Vetor.

Conselho Federal de Psicologia (2003). Resolução CFP 02/2003. Brasília: Conselho Federal de Psicologia.

Cordás, T. A. \& Neves, J. E. P. (1999). Escalas de avaliação em transtornos alimentares. Revista de Psiquiatria Clínica, 26(1), 41-47.

Cunha, J. A. (2000). Técnicas projetivas gráficas: por que sim? por que não? São Paulo: Casa do Psicólogo.

Freitas, S., Gorenstein, C. \& Appolinário, J. C. (2002). Instrumentos para avaliação dos transtornos alimentares. Revista Brasileira de Psiquiatria, 24(3), 34-38.

Güntert, A. E. V. A. (2000). Técnicas projetivas: o geral e o singular em avaliação psicológica. Em F. F. Sisto, E. T. B. Sbardelini \& R. Primi (Orgs.). Contextos e questôes da avaliação psicológica (pp. 77-84). São Paulo: Casa do Psicólogo.

Justo, H. \& Van Kolck, T. (1996). O Teste das Pirâmides de Cores. São Paulo: Vetor. 
Murray, H. A. (2005). Teste de Apercepção Temática (M. C. V. M. Silva, trad.). São Paulo: Casa do Psicólogo.

Nijamkin, G. C. \& Braude, M. G. (2000). O Questionário Desiderativo (L. S. P. C. Tardivo, trad.). São Paulo: Vetor.

Ocampo, M. L. S. \& Arzeno, M. E. G. (1981a). Força e fraqueza da identidade no Teste Desiderativo (M. Felzenszwalb, trad.). Em M. L. S. Ocampo, M. E. G. Arzeno \& E. G. Piccolo (Orgs.). O processo psicodiagnóstico e as técnicas projetivas (pp. 203-311). São Paulo: Martins Fontes.

Ocampo, M. L. S. \& Arzeno, M. E. G. (1981b). O Teste de Relações Objetais de Herbert Phillipson (M. Felzenszwalb, trad.). Em M. L. S. Ocampo, M. E. G. Arzeno \& E. G. Piccolo (Orgs.). O processo psicodiagnóstico e as técnicas projetivas (pp. 101-145). São Paulo: Martins Fontes.

Oliveira, E. A. \& Santos, M. A. (2006). Perfil psicológico de pacientes com anorexia e bulimia nervosas: a ótica do psicodiagnóstico. Medicina, 39(3), 353-360.

Peres, R. S. \& Justo, J. S. (2005). Contribuições das técnicas projetivas gráficas para a compreensão da personalidade de andarilhos de estrada. Estudos de Psicologia (Natal), 10(2), 305-312.

Peres, R. S. \& Santos. M. A. (2006). Contribuições do Desenho da Figura Humana para a avaliação da imagem corporal na anorexia nervosa. Medicina, 39(3), 361-370.

Peres, R. S., Santos, M. A. \& Kruschewsky, M. S. L. B. (2007). Imagem corporal em obesos mórbidos antes e depois de uma intervenção multidisciplinar. Psychologica, 44, 467-88.
Rosa, J. T. \& Silva, J. C. V. V. V. (2005). Fundamentos do Teste de Phillipson na prática clínica. Em J. T. Rosa \& J. C. V. V. V. Silva (Orgs.). Desenvolvimentos na prática clínica com o Teste de Phillipson (pp. 17-25). São Paulo: Vetor.

Silva, J. C. V. V. V. \& Paegle, I. C. M. (2006). Transtornos alimentares: funcionamento mental e sofrimento psíquico em pacientes com anorexia nervosa. Mudanças, 14(2), 186-196.

Sopezki, D. S. (2007). Relacionamento primário com a figura materna e auto-estima em mulheres com transtornos alimentares (Dissertação de Mestrado). Porto Alegre: Pontifícia Universidade Católica do Rio Grande do Sul - Faculdade de Psicologia.

Vaz, C. E. (2006). Contribuições do Rorschach como técnica projetiva na avaliação psicológica da personalidade. Em B. S. G Werlang \& M. S. Oliveira (Orgs.). Temas em Psicologia Clínica (pp. 151-153). São Paulo: Casa do Psicólogo.

Villemor-Amaral, A. E. (2005). As Pirâmides Coloridas de Pfister. São Paulo: CETEPP.

Werlang, B. S. G., Villemor-Amaral, A. E. \& Nascimento, S. R. G. F. (2010). Avaliação psicológica, testes e possibilidades de uso. Em Conselho Federal de Psicologia (Org.). Avaliação psicológica: diretrizes na regulamentação da profissão (pp. 87-100). Brasília: Conselho Federal de Psicologia.

Recebido em 02/04/2011

Reformulado em 01/07/2011

Aprovado em 01/08/2011

Sobre os autores:

Rodrigo Sanches Peres é psicólogo pela Universidade Estadual Paulista, mestre e doutor em Psicologia pela Universidade de São Paulo; especialista em Psicologia Clínica pelo Conselho Federal de Psicologia e professor do Instituto de Psicologia da Universidade Federal de Uberlândia.

Manoel Antônio dos Santos é psicólogo, mestre e doutor em Psicologia Clínica pela Universidade de São Paulo; professor da Faculdade de Filosofia, Ciências e Letras de Ribeirão Preto da Universidade de São Paulo e bolsista de produtividade em pesquisa do Conselho Nacional de Desenvolvimento Científico e Tecnológico.

Notas

1. Nas referidas consultas preliminares, as bases de dados adotadas foram PEPsic e SciELO-Brasil. Já os livros foram os seguintes: Psicodiagnóstico-V (ArtMed, 2000), Contextos e questões da avaliação psicológica (Vetor, 2000), Avaliação e medidas psicológicas: produção do conhecimento e da intervenção profissional (Casa do Psicólogo, 2002), Temas em avaliação psicológica (Casa do Psicólogo, 2005), Facetas do faz̧er em avaliação psicológica (Vetor, 2006), Avaliação psicológica: perspectivas e contextos (Vetor, 2007), Alguns domínios da avaliação psicológica (Alínea, 2007), Atualização em métodos projetivos para avaliação psicológica (Casa do Psicólogo, 2008). 
2. Os indicadores apresentados por Oliveira e Santos (2006) se referem essencialmente àqueles observados durante a realização da entrevista clínica empregada em conjunto com o Psicodiagnóstico de Rorschach, o TAT, o QD e o HTP no processo de avaliação psicológica reportado.

3. Os indicadores apresentados por Silva e Paegle (2006) se referem essencialmente àqueles observados durante a aplicação do TRO.

4. Os indicadores apresentados por Sopezki (2007), relacionados às respostas de movimento inanimado e aos fenômenos espaciais, se referem especificamente aos cartões I, VII e IX do Psicodiagnóstico de Rorschach. 